\title{
Genetic classification of languages examined or referred to
}

The following classification may not be definitive in every case. It is merely provided for readers' convenience.

Afro-Asiatic family: Arabic, Kambaata, Sidaama

Arawá family: Jarawara

Athabaskan family: Slavey

Austronesian family: Amis, Filipino, Hawaiian, Indonesian, Kapampangan, Kove, Lamaholot, Neku, 'Ôrôê, Tagalog, Tinrin

Chimbu-Wahgi (or Simbu) family: Dom

Chukchi-Kamchatkan family: Koryak

Dagestanian (or North East Caucasian) family: Avar

Dravidian family: Kurux, Malto

Eskimo-Aleut family: Iñupiaq

Hmong-Mien family: Hmyo

Indo-European family

Armenian branch: Armenian

Germanic branch: Danish, Dutch, English, German

Indo-Iranian branch: Bengali, Hindi, Maithili, Marathi, Nepali, Persian, Sadri, Sanskrit, Urdu

Italic branch: French, Portuguese, Spanish

Slavonic branch: Russian

Japonic family:

Japanese branch: Japanese

Ryukyuan branch: Miyako Ryukyuan (including Irabu Ryukyuan), Shuri (Okinawa) Ryukyuan, Yuwan (Amami) Ryukyuan

Kartvelian (or South Caucasian) family: Georgian

Mongolic family: Khalkha Mongolian

Niger-Congo family: Ewe, Godié, Leggbo, Matengo, Swahili

Pama-Nyungan family: Djaru, Wanyjirra, Warrongo

Sino-Tibetan family

Sinitic branch: Mandarin Chinese

Tibeto-Burman branch: Amdo Tibetan, Burmese, Central Tibetan, Chantyal, East Tibetan, Jinghpaw, Lahu, Meche, nDrapa, Newar, Pwo Karen, Sgaw Karen, Tiddim Chin

Tai-Kadai family: Lao, Thai

Tsimshianic family: Coast Tsimshian

Tungusic family: Manchurian, Nanay, Sive, Udihe, Ulcha

Turkic family: Sakha (Yakut), Turkish, Uzbek 
xiv - Genetic classification of languages examined or referred to

Yukaghir family: Kolyma Yukaghir, Tundra Yukaghir

Genetic affiliation not known for certain: Ainu, Korean

Not classifiable: Esperanto, Saramaccan, Tok Pisin 